\title{
Evaluating the effectiveness of eco-driving courses based on car-GPS tracking data in the itinerary tracking device to reduce fuel consumption of vehicles in urban areas
}

\author{
Van Manh $\mathrm{Do}^{1 *}$, Thi Lan Huong $\mathrm{Ho}^{1}$, and Tuan Hai Dinh ${ }^{2}$ \\ ${ }^{1}$ Civil Engineering Faculty, University of Transport and Communications, Hanoi, Vietnam. \\ ${ }^{2}$ Faculty of Urban Management, Hanoi Architectural University, Vietnam.
}

\begin{abstract}
According to the current issue of resource shortage, the reduction of fuel consumption or using alternative energies which has an environmentally friendly effect is the priority of governments in many countries. Besides, many studies mentioned that driver behaviors play an essential role to reduce fuel use and decrease vehicle exhaust emissions. On the other hand, the car- GPS tracking data in itinerary monitoring equipment is typically using in navigation, positioning, and vehicle management. Thus, this study aims to analyze the correlation between driver behaviors and fuel consumption based on the velocity, acceleration, fuel sensors data collected from car-GPS trackers device in association with Geographic Information System (GIS) to find out the solution to evaluate the effectiveness of ecodriving courses as well as assess the usability of using car-GPS tracking data from the GPS tracking device to analyze and adjust driver behavior in urban areas to save energy and protect the urban environment
\end{abstract}

\section{Introduction}

A few studies show that the driver's behavioral classification can be researched by two solutions, qualitative analysis, and quantitative analysis. While qualitative analysis by questionnaires with the participants of experts, scientists, and vehicle drivers still has certain limitations, the quantitative solution as analyzing the Jerk's profiles of driver behaviors based on speeding up or down of drivers is an efficient research method in many studies [1-5].

As mentioned above, nowadays, the data of the car GPS- tracking device is basically used for navigation and position purpose by managers, or for investigation of traffic accidents by police officers. There are few studies that utilized the data of car-GPS tracking devices to analyzed driver behaviors [6] and fuel consumption simultaneously [7, 8].

Moreover, the comprehensive process of analyzing driver behavior and fuel consumption should be provided by researchers or scientists to support urban citizens, city governments according to the valuable data of GPS-tracking device to reduce the fuel use as well as decrease the harmful impact of vehicle exhaust emission to the urban environment.

\section{Methodology}

\subsection{Driver's behavior analysis model based on car-GPS tracking data}

In this study, the authors propose classifying the driver's behavior by analyzing the variable acceleration in a car as a Jerk's profile for the drivers, together with calculating the

* Corresponding author: manhdv@,utc.edu.vn 
vehicle specific power (VSP) values before and after eco-driving courses. In general, the analytical process is as the following expression (Fig.1).

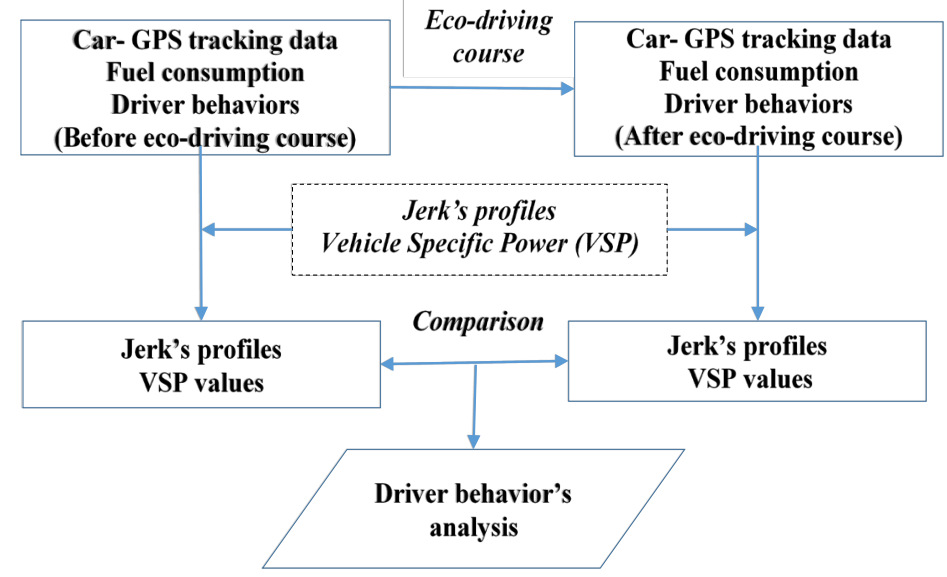

Fig. 1. Driver behavior's analysis model.

\subsection{Data collection}

Car-GPS tracking device also known as GPS tracker car tracking device, car itinerary tracking device, or car navigation device is usually about $5 \times 12 \mathrm{~cm}$ in size, firmly designed with a special metal case with anti-collision properties shockproof, withstand a high temperature of about 800 degrees Celsius. Basically, this device has good quality and can operate in many extreme weather conditions (Fig.2).

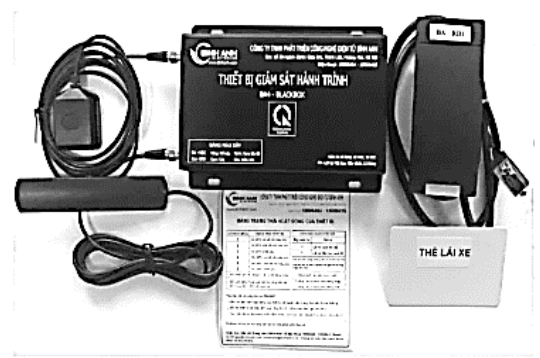

(a)

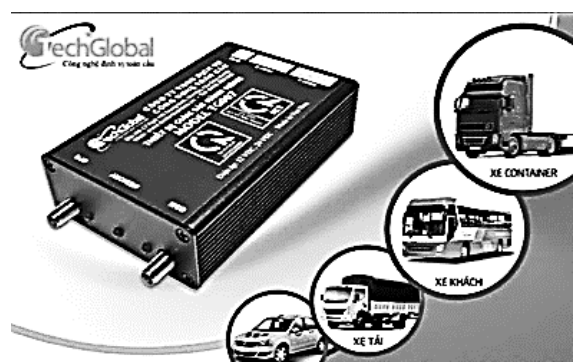

(b)

Fig. 2. The samples of the car GPS tracking devices.

The Car-GPS tracking device is professionally installed inside the vehicle and connects to the online management server system via SMS/ GPRS/ GPS, allowing users to manage their vehicle information through computers or phones that connected to the internet. All parameters of position, speed, acceleration, travel distance, parking stops, air condition, fuel consumption...etc are displayed on the computer screen of the manager 24/24 hour. All information and data of the vehicle are normally stored on the server during the last 3 months (Fig.3). 


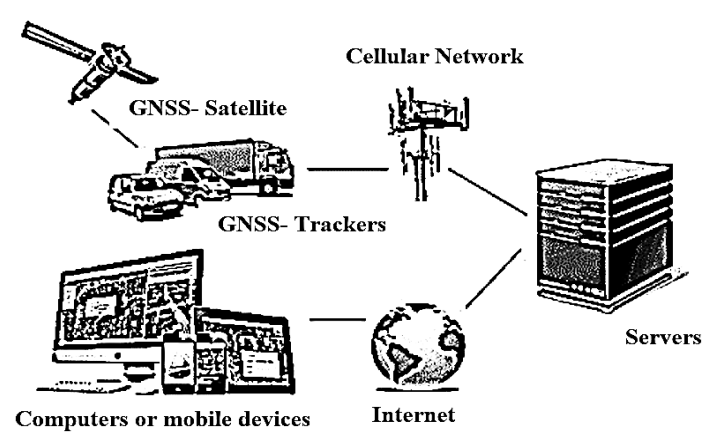

Fig. 3. Real time car-GPS tracking system [9].

In Vietnam, vehicles registered for service or commercial operation of transport enterprises, when going to be registered, must be equipped with the car-GPS tracking device to operate the service in the road traffic system according to the National standard named QCVN 31:2014 / BGTVT [10] and National circular No. 09/ 2015 / TT-BGTVT [11], one of the Vietnamese government's efforts towards traffic safety and traffic efficiency.

Nevertheless, most of the car-GPS tracking data just used to analyze traffic accidents as well as managing vehicles in the road traffic network in Vietnam or some Asia countries.

Thus, in this study, we suggest that the vehicle speed, variable acceleration, and position based on car GPS-trackers, together with fuel consumption according to the fuel sensors, and the GIS data of the road network should be used to analyze the driver behaviors and fuel consumption simultaneously.

\subsection{Classify driver's behavior according to jerk's profiles and VSP values}

Basically, the driver's behavior classified through the way the driver impacts the vehicle by speeding up or down [1]. Briefly, the driving style (DS) could be classified into three levels basis as the following expression :

- DS1 (Calm driving): Drivers who predict traffic situations and other vehicle movements, pay more attention to traffic signals, and speed up or down slightly and smoothly. Therefore, this type of driving is the most fuel-efficient driving style.

- DS2 (Normal driving): In this type of driving classification, the driver usually has moderate behavior in acceleration, deceleration, and reducing speed (braking). Thus, this type of DS is also fuel-efficient style.

- DS3 (Aggressive driving): This type of aggressive driving is for those who have a temper and want to overcome obstacles in the fastest possible way, this driving style is often increased or decreased speed significantly, along with braking quickly. This driving style consumes the most energy.

As discussed above, the analysis of Jerk's profile could classify the driving style as a quantitative solution. In physical science, the Jerk or Yank is the rate of variation of the acceleration. Specifically, Jerk's value equals the first derivative of the acceleration, the quadratic derivative of the speed (velocity), and the cubic derivative of the coordinate increment during the computation time. Hence, Jerk's profile is defined by the following equation:

$$
\vec{j}=\frac{d \vec{a}}{d t}=\frac{d^{2} \vec{v}}{d t^{2}}=\frac{d^{3} \vec{r}}{d t^{3}}
$$

Where: $\mathrm{j}\left(\mathrm{m} / \mathrm{s}^{3}\right), a\left(\mathrm{~m} / \mathrm{s}^{2}\right), \mathrm{v}(\mathrm{m} / \mathrm{s}), \mathrm{r}(\mathrm{m})$ are the jerk's value, acceleration, velocity, and coordinate increment respectively. 
While graphical analysis of acceleration indicates accelerating or slowing down the driver, a graphical analysis of the variable speed of acceleration (jerk's profile) indicates the details of acceleration or deceleration action. The driver's instantaneous speed, this is a relatively powerful algorithm for determining the driver's behavior in a particular situation.

This analysis is quite common in the US's aerodynamic analysis standards such as UDDS (Urban Dynamometer Driving Schedule) [12-14] when there is a large variation in the acceleration, which has positive and negative values, then classify the driving style of drivers.

The most significant in analyzing the rate of variation of acceleration in Jerk's graph is that the maximum and minimum values of this graph appear only when there is a sudden variation in the value of the acceleration. It is one of the main factors affecting the fuel consumption of the engine. Therefore, calculating the Jerk's value not only classifies driving style but also simultaneously analyzes the fuel consumption.

In general, analyzing the driver's behavior according to quantitative methods as Jerk's profiles also shows limitations in analyzing behavior. In fact, the drivers express all three driving states as mentioned above in their journey. Then, the driving classification needs concerning the most common style based on the average value of jerk's profile for the entire journey. According to [1], we suggest the classification of driver is calculated by the following expression:

i) Step 1: Calculate the Jerk's profile as a formula (1) in specified time $\mathbf{J}(t), t \in$ $\left[\left(t_{c}-\omega\right), t_{c}\right], \omega$ is window size, tc is timing of consideration $\left(t_{c}=1,2,3 \ldots\right)$.

ii) Step2: Calculate the standard deviation of the jerk's profile for the whole experimental process as the following equation:

$$
S D_{j}=\sqrt{\frac{1}{n-1} \sum_{i=1}^{n}\left(j_{i}-\bar{j}\right)^{2}}
$$

Where: $\bar{J}$ is the average value of Jerk's profile, ji is the value of Jerk's feature for each time step analysis (window size).

iii) Step 3: Determine the actual type of road that the vehicle passes, the level of traffic congestion, then estimate the rated value $\overline{j_{a v}}$ (the average value of variable speed of the rated acceleration is calculated for each specific road type is based on GIS data and LOS (Level Of Service) according to [15] and our experiment analysis, We suppose the averge of Jerk value as the following expression (Table.1).

Table 1. The average Jerk values based on LOS and road type (GIS data)

\begin{tabular}{|c|c|}
\hline LOS or Road type & $\overline{\overline{\boldsymbol{j}}_{\boldsymbol{a v}}}\left(\mathbf{m} / \mathbf{s}^{\mathbf{3}}\right)$ \\
\hline LOS A & 0.2131 \\
\hline LOS B & 0.2126 \\
\hline LOS C & 0.2258 \\
\hline LOS D & 0.2075 \\
\hline LOS E & 0.2401 \\
\hline LOS F & 0.3096 \\
\hline Urban road & 0.2925 \\
\hline Rural road & 0.2439 \\
\hline
\end{tabular}

While LOS A represents the best-operating conditions, with low level traffic jams or congestion, LOS F represents a crowded route, with vehicles challenging to move. In the above calculation table, the geometry parameters of the road type have been detailed and calculated based on the geographic information data (GIS), the geometry of the road, and the driver's behavior. 
iv) Step 4: Calculate the Jerk feature

$$
J_{f}=\frac{S D_{j}}{\overline{j_{a v}}}
$$

v) Step 5: Compare

- If $J_{f}<0.5$ then this driving style is DS1

- If $0.5<J_{f}<1.0$ then this driving style is DS2

- If $J_{f}>1.0$ then this driving style is DS3

\subsection{Vehicle specific power (VSP)}

Several studies indicated the correlation between vehicle exhaust emission and fuel consumption to calculate the VPS value in different formulas based on the engine type, engine current operating system, ambient temperature, etc [16-18].

However, Fangfang Zheng, (2017)[3] and Song et al. (2015) [19] suggested a formula that stimulated the vehicle exhaust emissions and fuel consumption depending on velocity (v), acceleration (a) as the following equation:

$$
V S P=0.132 v+1.1 v \cdot a+0.0003202 v 3[\mathrm{~kW} / \mathrm{ton}]
$$

The above equation not only has a close relation to the physical process of driving but also could apply to calculate the VSP values according to GPS data, of vehicles in the GPS tracking devices.

\subsection{Eco-driving course}

In this study, the authors propose eco-driving techniques with 08 operating skills and 04 tips for drivers in the process of driving with specific skills are as following expressions [9]:

- 08 operating skills:

- Use the suitable gear number in the car, and move quickly to the highest gear possible to reduces engine revs.

- Keep a steady speed and distance, operate the vehicle within the engine's fuel-saving rotational speed and the vehicle's fuel-saving speed (eco-mode) according to the vehicle manufacturer's recommended information.

- $\quad$ Drive carefully, slowly start off, and speed up or down the vehicle.

- $\quad$ Trying to keep the vehicle moving on the road when transporting.

- Avoid heavy braking, use the slight and smooth braking and deceleration techniques.

- Avoid over-revving, predict traffic conditions to restrict sudden acceleration, deceleration, and fast braking.

- Turn off the electrics, minimize idling time, and unnecessary engine heating.

- Remove unnecessary baggage, do not overload the vehicle, decrease the vehicle's aerodynamic drag.

- 04 tips:

- Check the tires and maintaining appropriate tire pressure.

- Use the suitable fuel and lubricating oil, and properly use auxiliary equipment in the vehicle.

- $\quad$ Pay more attention to the inspection and maintenance of vehicles on time.

- Use fuel-efficient driving systems and equipment. 


\section{Implementation}

\subsection{Study area}

The study area is in Hanoi city, the capital of Vietnam, the experimental road starts from the My Dinh Stadium to Le Quang Dao street, then Thang Long Boulevard, go through the Tran Duy Hung street, the return point at the intersection named Tran Duy Hung and Lang $\mathrm{Ha}$ intersection, the total length of the experiment rout is more than $10 \mathrm{~km}$. According to the GIS data of the road network in Hanoi city, the Le Quang Dao street was congested due to the construction of the F1 race track. Additionally, in the Thang Long Boulevard, the vehicle moves easily, along with the intersection named Tran Duy Hung and Lang Ha intersection has a high density of traffic flows during the experimental time (Fig.4).

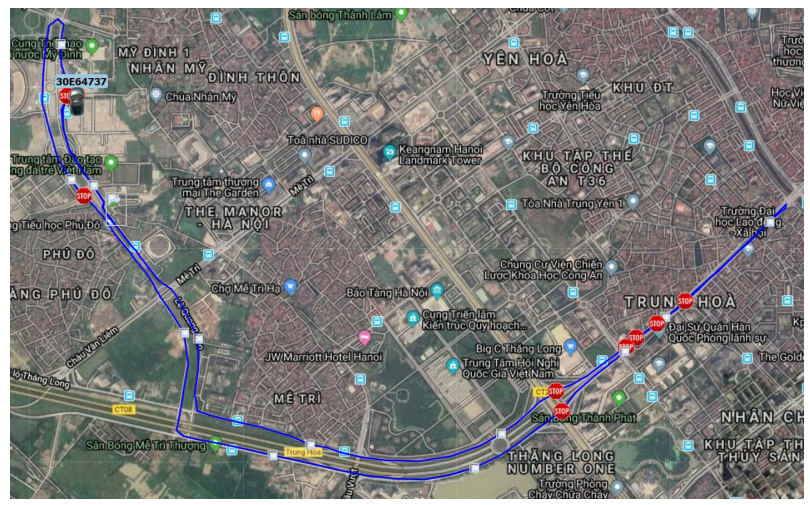

Fig. 4. Study area

\subsection{Result analysis}

After the experimental process on the same study area for both drivers before and after the eco-driving course, the coordinate and velocity data collected by the car-GPS tracking device, together with fuel consumption data were extracted and analyzed to calculate the Jerk's profile and VSP values.

The results show that the driver's behavior has an impact on the increase or decrease in fuel consumption after the eco-driving course, the fuel consumption decreased by 0.3 liters per $100 \mathrm{~km}$.

According to (section 2.3), $0.5<\mathrm{Jf}=0.514<1$ therefore the driving style of the driver is classified as DS2 before the eco-driving course. Additionally, the $\mathrm{Jf}=0.457<0.5$, therefore the driving style is classified as DS1 after the eco-driving course.

The experimental results show that the driver's behavior could be determined by the Jerk's profile based on the car-GPS tracking data and validated by the VSP values simultaneously (Table. 2).

Table. 2. Experimental results

\begin{tabular}{|c|c|c|c|}
\hline Factors & $\begin{array}{c}\text { Before eco-driving } \\
\text { course }\end{array}$ & \multicolumn{1}{|c|}{ After eco-driving course } & Difference \\
\hline Driver's behavior & $\begin{array}{c}\text { Driving according } \\
\text { to daily habits }\end{array}$ & $\begin{array}{l}\text { reduced. The process of speeding up or } \\
\text { down was softer and smoother, } \\
\text { allowing the engine to operate at the } \\
\text { recommended speed (eco-mode) }\end{array}$ & $\begin{array}{c}\text { Getting } \\
\text { better }\end{array}$ \\
\hline
\end{tabular}




\begin{tabular}{|c|c|c|c|}
\hline & & \multicolumn{1}{|c|}{$\begin{array}{l}\text { according to the manufacture's } \\
\text { instructions. }\end{array}$} & \\
\hline $\mathrm{SD}_{\mathrm{j}}$ & 0.107 & 0.099 & -0.008 \\
\hline$\overline{\overline{j a v}_{a v}}\left(\mathrm{~m} / \mathrm{s}^{3}\right)$ & \multicolumn{3}{|c|}{0.2075} \\
\hline $\mathrm{J}_{\mathrm{f}}$ & 0.514 & 0.475 & -0.038 \\
\hline $\mathrm{VSP}(\mathrm{KW} /$ ton$)$ & 1.030 & 0.892 & -0.138 \\
\hline $\begin{array}{c}\text { The average fuel } \\
\text { consumption per } \\
\text { 100km (L/100km) }\end{array}$ & 6.1 & 5.8 & -0.300 \\
\hline
\end{tabular}

\section{Conclusions and remarks}

After analyzing data based on car-GPS tracking equipment, car-fuel sensors, driver's behavior before and after the eco-driving course, the experimental results show that the driver's behavior was identified, analyzed, and improved.

This research has shown the correlation between driver behavior and fuel consumption according to the methodology and detailed analysis as mentioned above.

Furthermore, the comprehensive model to analyze driver behavior and fuel consumption simultaneously was provided by quantitative solutions ( analyzing the Jerk's profiles and calculating the VSP values) based on car-GPS tracking devices and GIS.

However, the number of test samples is limited. More studies are needed on researching the correlation between drivers' behavior and fuel consumption through the car-GPS tracking database obtained from the car-GPS tracking device, GPS data, CORS data (Continuosly Operation Reference Station data) to reduce fuel consumption and protect urban life.

\section{References}

1. Murphey, Y.L., R. Milton, and L. Kiliaris. Driver's style classification using jerk analysis. in 2009 IEEE Workshop on Computational Intelligence in Vehicles and Vehicular Systems (2009)

2. Ferreira, J.C., J.d. Almeida, and A.R.d. Silva, The Impact of Driving Styles on Fuel Consumption: A Data-Warehouse-and-Data-Mining-Based Discovery Process. IEEE Transactions on Intelligent Transportation Systems, 16(5): p. 2653-2662 (2015)

3. Zheng, F., et al., Influence of driver characteristics on emissions and fuel consumption. Transportation Research Procedia, 27: p. 624-63 (2017)

4. Feng, Y., et al. Driving Style Analysis by Classifying Real-World Data with Support Vector Clustering, in 2018 3rd IEEE International Conference on Intelligent Transportation Engineering (ICITE) (2018)

5. Zhao, Y., T. Yamamoto, and T. Morikawa, An analysis on older driver's driving behavior by GPS tracking data: Road selection, left/right turn, and driving speed. Journal of traffic and transportation engineering (English edition), 5(1): p. 56-65 (2018)

6. Ceikute, V. and C.S. Jensen. Routing service quality local driver behavior versus routing services, in 2013 IEEE 14th International Conference on Mobile Data Management. IEEE (2013)

7. Berry, I.M., The effects of driving style and vehicle performance on the real-world fuel consumption of US light-duty vehicles, Massachusetts Institute of Technology (2010)

8. Beusen, B., et al., Using on-board logging devices to study the longer-term impact of an eco-driving course. Transportation research part D: transport and environment, 14(7): $\mathrm{p}$. 514-520 (2009)

9. Website: http://www.alhadeedtechnologies.com/howitworks.html. 
10. (Vietnam), M.o.T., National technical regulation on vehicle tracking equipment, Ministry of Transport of Vietnam, Vietnam (2014)

11. (Vietnam), M.o.T., Regulation on provision, management and use of data from vehicle tracking device. September, Ministry of Transport of Vietnam, Vietnam (2015)

12. Ding, X., et al., An improved Thevenin model of lithium-ion battery with high accuracy for electric vehicles. Applied Energy, 254: p. 113615 (2015)

13. Maia, R., et al. Electric vehicle simulator for energy consumption studies in electric mobility systems. in 2011 IEEE Forum on Integrated and Sustainable Transportation Systems, IEEE (2011)

14. Peterson, S.B., J. Apt, and J. Whitacre, Lithium-ion battery cell degradation resulting from realistic vehicle and vehicle-to-grid utilization. Journal of Power Sources, 195(8): p. 2385-2392 (2010)

15. Manual, H.C., Highway capacity manual. Washington, DC, 2 (2000)

16. Barth, M., et al., The development of a comprehensive modal emissions model, $\|$ NCHRP Web-Only Document 122. Contractor's final report for NCHRP Project, 2000: p. 25-11 (2000)

17. Jimenez-Palacios, J.L., Understanding and quantifying motor vehicle emissions with vehicle specific power and TILDAS remote sensing. Massachusetts Institute of Technology, 1998.

18. Scora, G. and M. Barth, Comprehensive modal emissions model (cmem), version 3.01. User guide. Centre for environmental research and technology. University of California, Riverside, 1070 (2006)

19. Song, G., L. Yu, and Z. Geng, Optimization of Wiedemann and Fritzsche car-following models for emission estimation. Transportation Research Part D: Transport and Environment, 34: p. 318-329 (2015) 